\title{
Sonic hedgehog signalling proteins are frequently expressed in retinoblastoma and are associated with aggressive clinicopathological features
}

\author{
Ji-Young Choe, ${ }^{1,2}$ Ji Yun Yun, ${ }^{1,2}$ Yoon Kyung Jeon, ${ }^{1,3}$ Se Hoon Kim, ${ }^{4}$ \\ Ho-Kyung Choung, ${ }^{5}$ Sohee Oh, ${ }^{6}$ Mira Park, ${ }^{7}$ Ji Eun Kim ${ }^{1,7}$
}

\begin{abstract}
${ }^{1}$ Department of Pathology, Seoul National University, College of Medicine, Seoul, Korea

${ }^{2}$ Department of Pathology, Seoul National University Bundang Hospital, GyeonggiDo, Korea

${ }^{3}$ Department of Pathology,

Seoul National University

Hospital, Seoul, Korea

${ }^{4}$ Department of Pathology,

Yonsei Unversity, College of

Medicine, Seoul, Korea

${ }^{5}$ Department of

Ophthalmology, Seoul National

University Boramae Hospital, Seoul, Korea

${ }^{6}$ Department of Biostatistics, Seoul National University Boramae Hospital, Seoul, Korea

${ }^{7}$ Department of Pathology, Seoul National University Boramae Hospital, Seoul, Korea
\end{abstract}

\section{Correspondence to} Dr Ji Eun Kim,

Department of Pathology, Seoul National University Boramae Hospital, 20, Boramae-Ro 5-Gil, DongjakGu, Seoul 110-744, Korea; npol181@snu.ac.kr

Received 21 May 2014 Revised 29 August 2014

Accepted 20 September 2014 Published Online First

8 October 2014

\section{CrossMark}

To cite: Choe J-Y, Yun JY, Jeon YK, et al. J Clin Pathol 2015;68:6-11.

\section{ABSTRACT}

Aims This study aimed to examine the expression of Sonic hedgehog (SHH) signalling proteins in retinoblastoma and to evaluate its clinical significance. Methods Seventy-nine enucleated retinoblastoma tumours were investigated immunohistochemically using antibodies against $\mathrm{SHH}$ pathway proteins, such as $\mathrm{SHH}$, glioma-associated oncogene homologue (GLI) 1, GLI2, $G L 13$ and $A B C$ binding cassette $G 2$ (ABCG2). Western blotting of SHH signalling proteins was performed in two retinoblastoma cell lines.

Results $\mathrm{SHH}$ was expressed in most retinoblastoma cases (78 of 79,98.7\%), with 21 cases (26.6\%)

showing strong expression. GLI1 and GLI2 were also frequently expressed: 67 of 78 cases (85.9\%) and 71 of 77 cases $(92.2 \%)$, respectively. GLI3, a transcriptional repressor, was expressed at low levels in 23 of the 78 cases (29.5\%). High ABCG2 expression was found in 23 of the 78 cases $(29.5 \%)$. High expression levels of these proteins in retinoblastoma cell lines were confirmed by western blotting. The expression of SHH was associated with advanced stages, local invasion and metastasis (all $\mathrm{p}<0.05$ ).

Conclusions $\mathrm{SHH}$ signalling molecules were frequently expressed in retinoblastoma tumour cells, and high $\mathrm{SHH}$ expression was closely related to an advanced disease status. Our results suggest that the SHH signalling pathway may play a role in the progression of retinoblastoma.

\section{INTRODUCTION}

Retinoblastoma is the most common primary intraocular malignancy of infancy and childhood, especially before the age of 5 years. ${ }^{1}$ The incidence of retinoblastoma has been estimated between 1:30000 and 1:15 000 live births per year. ${ }^{2}{ }^{3}$ The pivotal genetic event in the pathogenesis of retinoblastoma is the inactivation of both alleles of the retinoblastoma gene $(R B)$, which is a regulator at the cell-cycle checkpoint between the G1 and S phases. ${ }^{4}$ However, little is known about additional pathological alterations regarding the biological aggressiveness of the disease, aside from the silencing of the $R B \cdot{ }^{4-6}$ Chromosomal aberrations, such as an increase in copy number of $6 \mathrm{p}$ and $1 \mathrm{q}$ or loss of $1 \mathrm{p}$, inactivation of tumour suppressor genes, such as P53, and epigenetic mechanisms, such as aberrant promoter methylation of $p 16 I N K 4 A$, have been found in only a small subset of retinoblastoma. ${ }^{78}$ Despite significant research, there are few universally acceptable biomarkers to identify the risk of tumour progression or to predict clinical outcomes in retinoblastoma.

Sonic hedgehog (SHH), a vertebrate homologue of the Drosophila segment polarity gene product hedgehog, is a secreted protein that plays a critical role in cell proliferation and differentiation during embryonic development. ${ }^{6910}$ Activation of SHH signalling is mediated by the glioma-associated oncogene homologue (GLI) transcription factors (GLI1, 2 and $3)$, which trigger several downstream cascade pathways, including multidrug resistant pumps, such as ATP-binding cassette G2 (ABCG2) ${ }^{11-13}$ Recently, the SHH signalling pathway has been re-emphasised in terms of cancer biology.

Dysregulation of the SHH pathway has been reported in a broad range of cancers, most notably in basal cell carcinoma and medulloblastoma. ${ }^{14} 15$ However, very few attempts have been made at determining the role of $\mathrm{SHH}$ signalling in retinoblastoma. Based on the finding that $\mathrm{SHH}$ plays an important role in the differentiation of many embryonic organs, particularly of the retina, we hypothesised that the SHH pathway could be closely involved in the development or progression of retinoblastoma. In this study, we examined the expression profile of $\mathrm{SHH}$ signalling proteins in retinoblastoma tumour tissues and the normal retina, and correlated these with various clinicopathological parameters.

\section{METHODS}

Seventy-nine consecutive cases of retinoblastoma with enucleation between 1999 and 2011 were enrolled from the Seoul National University Boramae Hospital and the Seoul National University Hospital. Disease recurrence or progression was evaluated by funduscopic examination. The pathology of retinoblastoma was reviewed by two experienced pathologists. The patients were classified according to both the international retinoblastoma staging system and the American Joint Committee on Cancer (AJCC) staging system. ${ }^{16}{ }^{17}$ One pair of tissue microarray (TMA) blocks was constructed, each containing 79 tissue cores of $2 \mathrm{~mm}$ diameter, sampled from the most representative area of the donor blocks.

Immunohistochemistry (IHC) was performed using the BenchMark automatic immunostaining device (Ventana Medical Systems, Tucson, Arizona, USA) with validated protocols. Heat-induced epitope retrieval was performed using the Ventana CC1 mild reagent for $60 \mathrm{~min}$. Endogenous peroxidase was blocked by incubation with $0.3 \%$ 
hydrogen peroxide. After treatment with $10 \%$ normal goat serum to block non-specific antibody binding, immunohistochemical staining against the antibodies listed in table 1 was performed.

The interpretation of IHC was also performed by two pathologists. The results of SHH, GLI1 and GLI2 stainings were considered positive if $10 \%$ or more of the tumour cells were stained. ${ }^{14}$ The expression of SHH was scored as negative, low, intermediate or high and the expression of GLI1 and GLI2 was scored as negative, low or high, depending on the signal intensity. GLI3 was expressed as the percentage of positive tumour cells because the intensity was so homogeneous. Expression of ABCG2 in the tumour cells was compared with that observed in endothelial cells: if equal or higher, then the expression of ABCG2 was considered high; if lower, then the expression of ABCG2 was considered low. ${ }^{13}$ Appropriate internal or external controls were used for each antibody.

To validate the results of IHC, western blotting was performed in two retinoblastoma cell lines, Y79 and WERI-Rb-1 (both from American Type Culture Collection, ATCC, Manassas, Virginia, USA), with the same antibodies used for IHC. Cells in log-phase growth were collected, washed in cold phosphate buffered saline and lysed at $4^{\circ} \mathrm{C}$ in modified radioimmunoprecipitation assay buffer. An equivalent amount of cell lysate $(50 \mu \mathrm{g}$ of protein) resolved by $10 \%$ sodium dodecyl sulfate-polyacrylamide gel electrophoresis was transferred to polyvinylidene difluoride membranes. Non-specific binding was blocked by incubation in Tris-buffered saline supplemented with $0.1 \%$ Tween-20 (TBST) and 5\% non-fat skimmed milk. The blot was incubated with primary antibodies against SHH, GLI1, GLI2 and GLI3 in TBST containing 5\% bovine serum albumin at $4^{\circ} \mathrm{C}$ for $16 \mathrm{~h}$. The membrane was then washed with TBST and probed with the horseradish peroxidase-conjugated secondary antibody for $2 \mathrm{~h}$ at room temperature. The protein-antibody complexes were detected using enhanced chemiluminescence reagents (Amersham, Arlington Heights, Illinois, USA) according to the manufacturer's recommended protocol. $\beta$-Actin (Santa Cruz) was used as an internal positive control.

A statistical analysis was performed using IBM SPSS Statistics, V.20.0, for Windows (IBM, New York, USA). Pearson's $\chi^{2}$ test, Fisher's exact test or likelihood ratio test for trend was used to assess the associations between each protein expression levels and clinicopathological variables. Statistical significance was

Table 1 Antibodies used for immunohistochemistry

\begin{tabular}{|c|c|c|c|c|}
\hline Antibodies & Clone & Manufacturer & Localisation & $\begin{array}{l}\text { Positive } \\
\text { control }\end{array}$ \\
\hline SHH & H-160 & $\begin{array}{l}\text { Santa Cruz, } \\
\text { Santacruz, California, } \\
\text { USA }\end{array}$ & Cytoplasm & Endothelia \\
\hline GLI1 & $\mathrm{H}-300$ & $\begin{array}{l}\text { Santa Cruz, } \\
\text { Santacruz, California, } \\
\text { USA }\end{array}$ & $\begin{array}{l}\text { Nuclei and } \\
\text { cytoplasm }\end{array}$ & $\begin{array}{l}\text { Glioma } \\
\text { cells }\end{array}$ \\
\hline GLI2 & Polyclonal & $\begin{array}{l}\text { Abcam, Cambridge, } \\
\text { Massachusetts, USA }\end{array}$ & $\begin{array}{l}\text { Nuclei and } \\
\text { cytoplasm }\end{array}$ & $\begin{array}{l}\text { Glioma } \\
\text { cells }\end{array}$ \\
\hline GLI3 & $\mathrm{H}-280$ & $\begin{array}{l}\text { Santa Cruz, } \\
\text { Santacruz, California, } \\
\text { USA }\end{array}$ & $\begin{array}{l}\text { Nuclear } \\
\text { membrane }\end{array}$ & Endothelia \\
\hline$A B C G 2$ & BXP-21 & $\begin{array}{l}\text { Abcam, Cambridge, } \\
\text { Massachusetts, USA }\end{array}$ & Cytoplasm & Endothelia \\
\hline
\end{tabular}

defined as $p$ value $<0.05$. This study was approved by Institutional Review Board of the Seoul National University Boramae Hospital (reference number: 06-2011-157) on July 2011.

\section{RESULTS}

\section{Clinical characteristics}

The clinical findings of the 79 patients are summarised in table 2. Mutation of the $R B$ gene was examined in 20 patients using peripheral blood samples, revealing four patients with a mutated $R B$ in germ-line status. Nineteen patients received preoperative chemotherapy and nine of them were resistant to chemotherapy. The mean follow-up period of the patients was 67.4 months (median 69.1 months, range 1-144). Six patients had nodal or distant metastases at the time of enucleation; lymph nodes (1) and leptomeningeal involvement (5). Two patients developed recurrent mass at periorbital soft tissue and metastasis to bone marrow about 2 months (1) and 4 months (1) after enucleation and adjuvant chemotherapy.

\section{SHH signalling proteins in normal retina}

To determine the baseline expression of SHH-related proteins in a normal retina, we investigated the expression levels of $\mathrm{SHH}$, GLI1, GLI2 and GLI3 in an unaffected portion of enucleated

Table 2 Characteristics of patients with retinoblastoma

\begin{tabular}{|c|c|c|}
\hline \multirow[b]{2}{*}{ Variables } & \multicolumn{2}{|c|}{ Number of patients } \\
\hline & n (Total=79) & Per cent \\
\hline \multicolumn{3}{|l|}{ Age at diagnosis (months) } \\
\hline Median (range) & 22.2 & $(1-99)$ \\
\hline \multicolumn{3}{|l|}{ Age at operation (months) } \\
\hline Median (range) & 25.9 & $(1-113)$ \\
\hline Male sex & 44 & 55.7 \\
\hline Family history of RB & 2 & 2.5 \\
\hline Multifocal tumours & 6 & 7.6 \\
\hline Bilateral tumours & 18 & 22.8 \\
\hline \multicolumn{3}{|l|}{ International RB stage } \\
\hline Stage I & 73 & 92.4 \\
\hline Stage II & 0 & 0.0 \\
\hline Stage III & 0 & 0.0 \\
\hline Stage IV & 6 & 7.6 \\
\hline \multicolumn{3}{|l|}{ Pathological tumour stage } \\
\hline pT1 & 35 & 44.3 \\
\hline pT2 (a:b) & $23(11: 0)$ & 29.1 \\
\hline pT3 (a:b) & $17(11: 6)$ & 21.5 \\
\hline pT4 (a:b) & $3(1: 2)$ & 3.8 \\
\hline Unclassifiable & 1 & 1.3 \\
\hline Optic nerve invasion & 32 & 41.0 \\
\hline Choroidal invasion & 24 & 30.8 \\
\hline Extraocular invasion & 12 & 15.2 \\
\hline Nodal or distant metastasis & 6 & 7.6 \\
\hline \multicolumn{3}{|l|}{ Histopathological features } \\
\hline Necrosis & 62 & 78.5 \\
\hline Calcification & 59 & 78.7 \\
\hline Differentiation & 45 & 63.4 \\
\hline \multicolumn{3}{|l|}{ Further treatment } \\
\hline Preop. chemoradiotherapy & 19 & 24.1 \\
\hline Preop. local/proton therapy & 10 & 12.7 \\
\hline Postop. chemoradiotherapy & 21 & 26.6 \\
\hline
\end{tabular}



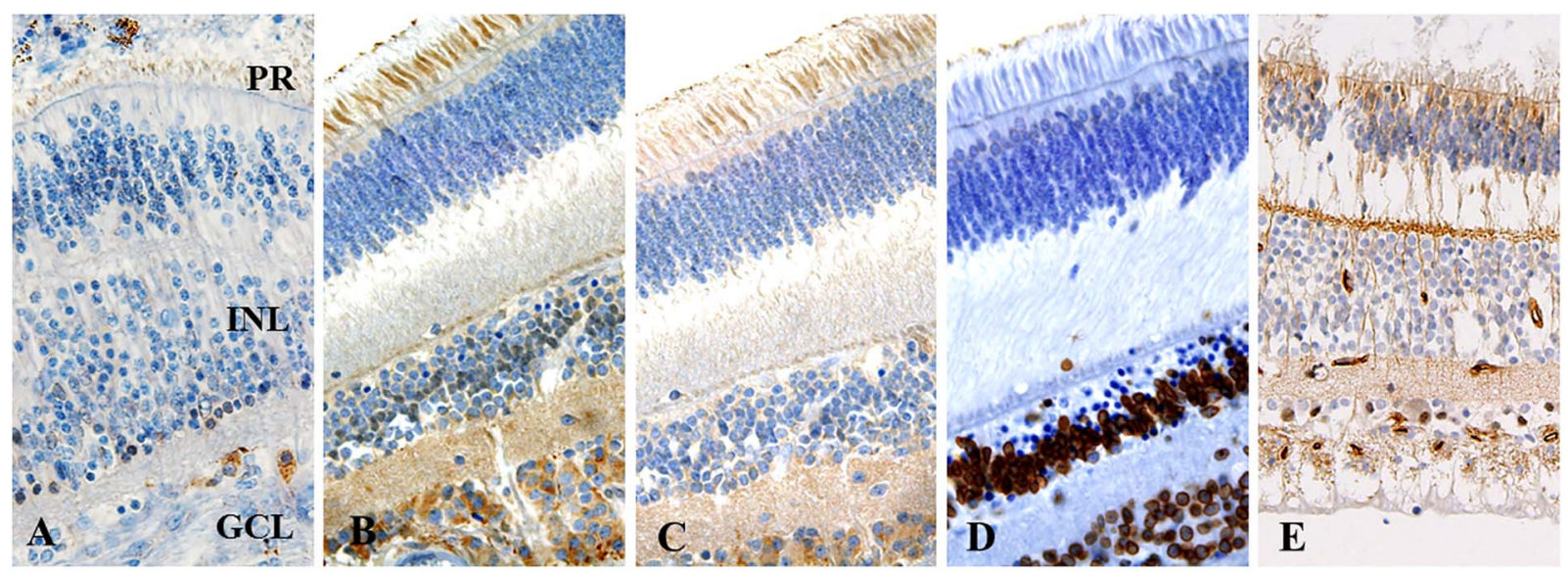

Figure 1 Expression of SHH signalling proteins in the normal retina. SHH (A), GLI1 (B), GLI2 (C) and ABCG2 (E) were expressed in the ciliated processes of PR cells. (A) SHH was rarely expressed in normal retina. (B, C) GLI1 was focally positive in the INL, and GLI1 and GLI2 were positive in the cytoplasm of the GCL. (D) GLI3 was expressed only in the stromal cells of the whole layer. (E) ABCG2 was positive in the vascular endothelial cells within the retina. $A B C G 2, A B C$ binding cassette $G 2 ; G L I$, glioma-associated oncogene homologue; SHH, Sonic hedgehog; GCL, ganglion cell layer; PR, photoreceptor; INL, inner nuclear layer.

specimens. In the normal retina, we detected SHH, GLI1, GLI2 and ABCG2 expression in the ciliated processes of photoreceptor cells but not in the cytoplasm. GLI1 was also focally expressed in the inner nuclear layer. The cytoplasmic staining of GLI1 and GLI2 appeared in the ganglion cell layer. GLI3 was strongly and diffusely expressed in the stromal cells in the whole layer (figure 1).

\section{SHH signalling proteins in retinoblastoma}

In the retinoblastomas, $\mathrm{SHH}$ was found in the cytoplasm of tumour cells in the majority of cases $(78 / 79,98.7 \%)$, and high expression was found in 21 cases (26.6\%). In contrast to the polarised and restricted expression in the cilia of the normal retina, the expression of SHH showed a variegated pattern in the tumour cells, with a diffuse, scattered granular or radiating pattern from the centre of florets or rosettes. The GLI1 and GLI2 proteins were expressed in the nuclei of tumour cells in 67 of 78 cases (85.9\%) and 71 of 77 cases (92.2\%), respectively. Strong GLI1 and GLI2 expression was noted in $10(12.8 \%)$ and 13 cases (16.9\%), respectively. GLI3 reactivity was found in the nuclear membrane, and the frequency of GLI3-positive cells ranged from $0 \%$ to $60 \%$ (median $0 \%$ ). Cases with more than $4 \%$ of GLI3 positivity were found in 23 of the 78 cases (29.5\%). Cytoplasmic ABCG2 was highly expressed in 23 of 78 cases $(29.5 \%$; figure 2$)$.

Two retinoblastoma cell lines, Y79 and WERI-Rb-1, showed expression of the SHH, GLI1, GLI2 and GLI3 proteins by western blotting, which confirmed the immunohistochemical results (figure 3). The expression of GLI3 was relatively weak compared with the other proteins.

\section{SHH signalling proteins and differentiation}

Differentiation of retinoblastoma was assessed by histological features. Each retinoblastoma was categorised as differentiated or undifferentiated tumours based on the presence of florets or rosettes and other cytological characteristics. The differentiated area with florets or rosettes was detected in 45 of the 71 cases (63.4\%).

Although histological evidence of differentiation was found focally in some cases, SHH expression was found diffusely in the majority of the cases, regardless of the histological diversity (figure 4). The expression of GLI1, GLI2 and ABCG2 proteins was significantly associated with undifferentiated tumours ( $p=0.016, p=0.043$ and $p=0.024$, respectively). In addition, differentiated tumours were associated with early age at enucleation $(\mathrm{p}=0.007)$, which coincides with previous studies. ${ }^{18}$ Undifferentiated tumours showed a tendency of frequent extraocular invasion $(\mathrm{p}=0.085)$, metastasis $(\mathrm{p}=0.002)$ and high $\mathrm{pT}$ stage by AJCC $(\mathrm{p}=0.063)$.

\section{SHH signalling proteins with clinicopathological features}

$\mathrm{SHH}$ expression was related to the extension of the tumour, including optic nerve invasion $(\mathrm{p}=0.004)$, choroidal invasion $(\mathrm{p}=0.023)$, extraocular inavasin $(\mathrm{p}=0.036)$, metastasis $(\mathrm{p}=0.001), \mathrm{pT}$ stage by AJCC $(\mathrm{p}=0.029)$ and the international retinoblastoma stage $(\mathrm{p}=0.001$; table 3$)$. High expression of $\mathrm{SHH}$ was also associated with absence of preoperative local or proton therapy $(\mathrm{p}=0.021)$. However, the expression of $\mathrm{SHH}$ signalling proteins was not different between patients with or without adjuvant chemotherapy. SHH expression showed positive correlations with GLI2 $(\mathrm{p}=0.012)$ and ABCG2 expression $(\mathrm{p}=0.019)$.

GLI1 expression was significantly high in bilateral tumours $(p=0.004)$ and was associated with early age of enucleation $(p=0.029)$. GLI2 expression was also associated with early age of diagnosis $(p=0.032)$, GLI1 expression $(p \leq 0.001)$ and high ABCG2 expression $(p=0.007)$. Survival analysis was not performed because there was no mortality and only two recurrences during the follow-up in our series.

\section{DISCUSSION}

This is the first study to investigate the expression of SHH signalling proteins and to evaluate their clinical significance in a large number of retinoblastoma samples. In this study, we found that SHH was expressed diffusely in the cytoplasm of tumour cells, whereas the normal retina showed expression restricted to the cilia of photoreceptor cells. Moreover, high SHH expression was associated with advanced disease status and adverse clinical outcomes. Our findings suggest that dysregulation of SHH signalling could be closely involved in the pathogenesis of retinoblastoma. 


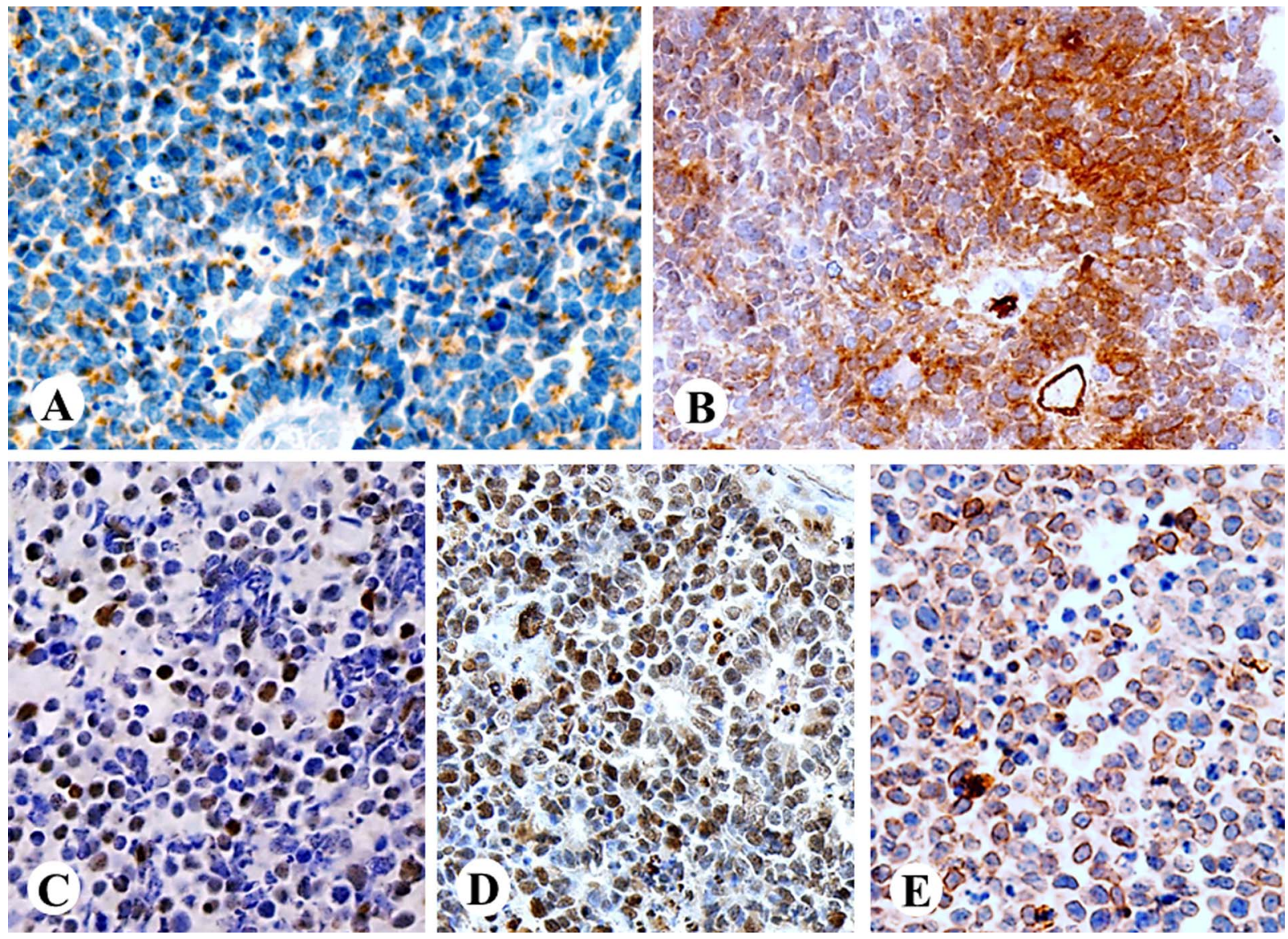

Figure 2 Retinoblastoma tumour cells show frequent high expression of SHH signalling proteins. (A) SHH was expressed in the tumour cell cytoplasm in the majority of the cases. (B) Strong expression of ABCG2 was found in some cases. (C, D) Diffuse nuclear and cytoplasmic positivity for GLI1 (C) and GLI2 (D) were found in many cases. (E) However, GLI3 was focally positive in a limited number of cases. ABCG2, $A B C$ binding cassette G2; GLI, glioma-associated oncogene homologue; SHH, Sonic hedgehog.

The role of the $R B$ gene in the development of retinoblastoma has been well established. However, the development of retinoblastoma requires additional molecular alterations to achieve a fully malignant phenotype. ${ }^{3} 619$ The majority of retinoblastomas involve at least one other genetic change in addition to the inactivation of $R B .^{5}{ }^{6} 20-23$ Dysregulation of $\mathrm{SHH}$ signalling is one of the possible candidates of an additional pathological alteration needed for retinoblastoma development. SHH signalling is involved in the development of a normal retina, and the loss of $\mathrm{SHH}$ expression has been found in dysplastic retinas in trisomy $13 .{ }^{24}$ In contrast to the well-polarised, strict localisation in the cell processes of normal photoreceptor cells, SHH expression in retinoblastoma showed a variegated pattern that suggested aberrant expression. Our results support that $\mathrm{SHH}$ is involved in the differentiation of tumour cells and that dysregulation of $\mathrm{SHH}$ signalling is linked to the pathogenesis of retinoblastoma.

Although retinoblastoma shares several clinicopathological characteristics with medulloblastoma, our findings suggest that the mechanism of $\mathrm{SHH}$ signalling could be different to that of medulloblastoma, with the latter displaying ligand-independent regulation. In a portion of medulloblastomas and basal cell carcinomas, $\mathrm{SHH}$ signalling is constantly activated without the binding of $\mathrm{SHH}$ ligand due to an activating mutation of the $\mathrm{SHH}$ receptor; thus, the expression of $\mathrm{SHH}$ in tumour cells can be observed in only a few cases. ${ }^{15}$ In our study, $\mathrm{SHH}$ expression was found in almost all the cases, regardless of differentiation or extent of the disease. These findings suggest that, in contrast to medulloblastoma, regulation of the $\mathrm{SHH}$ pathway in retinoblastoma is $\mathrm{SHH}$ ligand dependent and that $\mathrm{SHH}$ dysregulation might occur in the early stage of tumourigenesis.

Two types of ligand-driven $\mathrm{SHH}$ pathways are reported in the literature: paracrine and autocrine signalling. Paracrine signalling has been found in a subset of epithelial cancers, including colon, pancreatic and ovarian cancers. ${ }^{25}$ In the paracrine pathway, SHH protein secreted from tumour cells or stromal cells regulates the microenvironment and stimulates immune cells. However, autocrine signalling of the $\mathrm{SHH}$ pathway, in which the source of $\mathrm{SHH}$ is the tumour cell itself, has been observed in small-cell lung cancer and in some types of malignant lymphomas. ${ }^{26} 27$ We demonstrated SHH expression in retinoblastoma tumour cells by immunohistochemistry and in cell lines by western blotting. Our results support that the regulatory mechanism of $\mathrm{SHH}$ signalling in retinoblastoma may be ligand dependent, which is different from that of medulloblastoma. However, whether the mode of $\mathrm{SHH}$ activation in retinoblastoma is autocrine or paracrine remains unclear at this time.

We demonstrated the association of $\mathrm{SHH}$ protein expression and advanced disease status. Tumours with high expression of $\mathrm{SHH}$ ligand showed frequent local invasion, metastasis and advanced stages of both the international retinoblastoma staging system and the AJCC staging system. In addition, patients with high expression of GLI1 and GLI2 were diagnosed with retinoblastoma and underwent enucleation at earlier age. In contrast, 


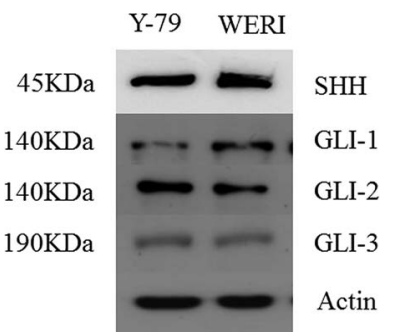

Figure 3 Western blotting of SHH signalling proteins in retinoblastoma cell lines. Two retinoblastoma cell lines, Y79 and WERI-Rb-1, show SHH, GLI1, GLI2, and GLI3 expression by western blotting. The expression of GLI3 was relatively weak compared with other proteins. GLI, glioma-associated oncogene homologue; SHH, Sonic hedgehog.

the expression of GLI3 and ABCG2 was not linked to the patients' clinical features. This lack of correlation between GLI3 and clinical features can be partially explained by the diverse functions of GLI proteins. Although all of the proteins examined in our study are known to be transcription factors or a direct downstream target of the $\mathrm{SHH}$ pathway, ${ }^{28}$ the expression or activation of GLI proteins is controlled by negative feedback between them. Hyperactivated GLI proteins can be suppressed by other GLIs, mainly by GLI3, a transcriptional repressor. ${ }^{29}$ Truncated, repressor form of GLI3 predominates in the quiescent hedgehog status. ${ }^{30}$ In our study, GLI3 expression was much lower compared with that of other SHH-related proteins, which was also confirmed by western blot assay in cell lines. These findings highly support the idea that $\mathrm{SHH}$ signalling is activated in retinoblastoma. We suspect that most nuclear GLI3 in tumour cells might be repressor form; however, this cannot be verified by immunohistochemistry.
Table 3 Association between expression of SHH signalling proteins and clinicopathological variables

\begin{tabular}{llllll}
\hline Variables ( $p$ value ${ }^{*}$ ) & SHH & GLI-1 & GLI-2 & GLI-3 & ABCG2 \\
\hline Age at diagnosis (<2 years) & 0.720 & 0.120 & $\mathbf{0 . 0 3 2}$ & 0.537 & 0.342 \\
Age at enucleation (<2 years) & 0.536 & $\mathbf{0 . 0 2 9}$ & 0.127 & 0.478 & 0.329 \\
Sex & 0.231 & 0.351 & $\mathbf{0 . 0 4 8}$ & $\mathbf{0 . 0 3 7}$ & 0.223 \\
Bilateral tumours & 0.460 & $\mathbf{0 . 0 0 4}$ & $\mathbf{0 . 0 1 4}$ & 0.388 & 0.623 \\
International RB stage & $\mathbf{0 . 0 0 1}$ & 0.581 & 0.446 & 0.240 & 0.240 \\
Pathological tumour stage & $\mathbf{0 . 0 2 9}$ & 0.541 & 0.264 & $\mathbf{0 . 0 2 4}$ & 0.748 \\
Optic nerve invasion & $\mathbf{0 . 0 0 4}$ & 0.786 & 0.634 & 0.163 & 0.509 \\
Choroidal invasion & $\mathbf{0 . 0 2 3}$ & 0.258 & 0.140 & 0.235 & 0.186 \\
Extraocular invasion & $\mathbf{0 . 0 3 6}$ & 0.683 & 0.541 & 0.091 & 0.503 \\
Nodal or distant metastasis & $\mathbf{0 . 0 0 1}$ & 0.581 & 0.446 & 0.240 & 0.240 \\
Differentiated tumour & 0.491 & $\mathbf{0 . 0 1 6}$ & $\mathbf{0 . 0 4 3}$ & 0.494 & $\mathbf{0 . 0 2 4}$ \\
Preop. chemoradiotherapy & 0.140 & 0.775 & 0.676 & 0.324 & 0.446 \\
Preop. local/proton therapy & $\mathbf{0 . 0 2 1}$ & 0.663 & 0.614 & 0.530 & 0.530 \\
Postop. chemoradiotherapy & $\mathbf{0 . 0 4 7}$ & 0.928 & 0.490 & 0.180 & 0.360
\end{tabular}

*Association was compared with Pearson's $\chi^{2}$ test or likelihood ratio test for trend. $A B C G 2, A B C$ binding cassette $G 2$; GLI, glioma-associated oncogene homologue; Postop, postoperative; Preop, preoperative; RB, retinoblastoma; SHH, Sonic hedgehog.

In addition, ABCG2 was frequently expressed in tumours and correlated closely to the expression of SHH-related proteins. ABCG2 is an ATP-dependent drug efflux pump that actively exports certain classes of chemotherapeutic drugs. In previous studies, ABCG2 was reported to be highly expressed in many invasive tumours, ${ }^{31}$ and the downregulation of ABCG2 by small interfering RNA reversed $\mathrm{SHH}$-induced chemoresistance. ${ }^{32}$ Although we could not find a direct association of ABCG2 with therapeutic responses in a small number of patients with chemotherapy, our study may point to the possibility of a link between $\mathrm{SHH}$ and
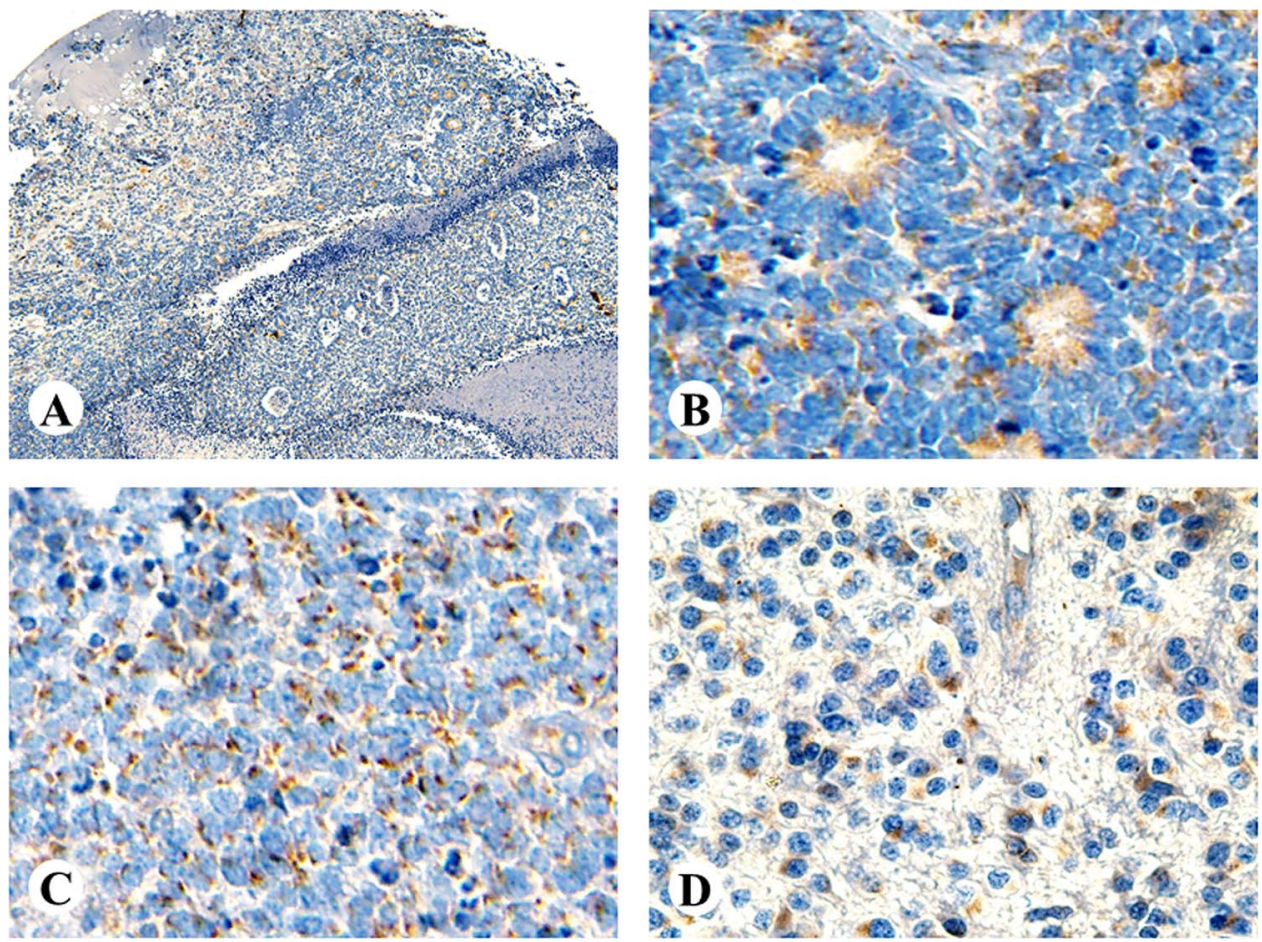

Figure 4 Immnohistochemical expression of Sonic hedgehog $(\mathrm{SHH})$ in the variously differentiated area of retinoblastoma. (A) SHH expression was observed in both differentiated and undifferentiated tumour areas: area of differentiation (B), undifferentiation (C) and differentiation with clear cytoplasm (D). 
therapeutic responses in retinoblastoma. Chemotherapy is the treatment of choice in retinoblastoma because of the strong desire to preserve the eye and due to improved responses. However, risk stratification with regard to relapse or persistent disease is still an unresolved task. Currently, several clinical trials of SHH pathway inhibitors are being conducted for various tumours, ${ }^{33}$ and we suggest that retinoblastoma is an additional candidate for SHH-targeted therapy.

In conclusion, we have shown that $\mathrm{SHH}$ signalling proteins are frequently expressed in the majority of retinoblastomas; furthermore, high expression of $\mathrm{SHH}$ can predict disease progression or adverse outcomes. These data provide a rationale for the further investigation of the SHH signalling pathway as a potential therapeutic target in retinoblastoma.

\section{Take home messages}

- Sonic hedgehog protein (SHH) was expressed in most cases of retinoblastoma tumours unlike normal retina.

- High expression of SHH was associated with advanced disease status including local invasion and metastasis.

- SHH signalling may participate in the pathogenesis or progression of retinoblastoma.

Contributors JEK and SHK designed and supervised this study. HKC and YKJ collected and analysed clinical characteristics of the patients. J-YC and JEK interpreted the pathological features and the immunohistochemistry. MP and JYY undertook experimental works. SO performed statistical analyses. J-YC wrote the paper. All authors reviewed the manuscript and approved the final article.

Funding This study was supported by a research fund from Seoul National University Boramae Hospital (03-2010-2) and partly supported by a research fund from Seoul National University Hospital (0520110070).

Competing interests None.

Ethics approval Institutional Review Board of the Seoul National University Boramae Hospital.

Provenance and peer review Not commissioned; externally peer reviewed.

\section{REFERENCES}

1 Mahoney MC, Burnett WS, Majerovics A, et al. The epidemiology of ophthalmic malignancies in new york state. Ophthalmology 1990;97:1143-7.

2 Fung YK, Murphree AL, T'Ang A, et al. Structural evidence for the authenticity of the human retinoblastoma gene. Science 1987;236:1657-61.

3 Poulaki V, Mukai S. Retinoblastoma: genetics and pathology. Int Ophthalmol Clin 2009;49:155-64.

4 Godbout R, Dryja TP, Squire J, et al. Somatic inactivation of genes on chromosome 13 is a common event in retinoblastoma. Nature 1983;304:451-3.

5 Kusnetsova LE, Prigogina EL, Pogosianz HE, et al. Similar chromosomal abnormalities in several retinoblastomas. Hum Genet 1982;61:201-4.

6 Wechsler-Reya R, Scott MP. The developmental biology of brain tumors. Annu Rev Neurosci 2001;24:385-428.

7 Indovina P, Acquaviva A, De Falco G, et al. Downregulation and aberrant promoter methylation of p16ink4a: a possible novel heritable susceptibility marker to retinoblastoma. J Cell Physiol 2010;223:143-50.
8 Gratias S, Schuler A, Hitpass LK, et al. Genomic gains on chromosome 1q in retinoblastoma: consequences on gene expression and association with clinical manifestation. Int J Cancer 2005;116:555-63.

9 Goodrich LV, Scott MP. Hedgehog and patched in neural development and disease. Neuron 1998;21:1243-57.

10 Hammerschmidt M, Brook A, McMahon AP. The world according to hedgehog. Trends Genet 1997;13:14-21.

11 Murone M, Rosenthal A, de Sauvage FJ. Sonic hedgehog signaling by the patched-smoothened receptor complex. Curr Biol 1999;9:76-84.

12 Singh RR, Kunkalla K, Qu C, et al. ABCG2 is a direct transcriptional target of hedgehog signaling and involved in stroma-induced drug tolerance in diffuse large B-cell lymphoma. Oncogene 2011;30:4874-86.

$13 \mathrm{Kim} J \mathrm{E}$, Singh RR, Cho-Vega JH, et al. Sonic hedgehog signaling proteins and atp-binding cassette g2 are aberrantly expressed in diffuse large b-cell lymphoma. Mod Pathol 2009;22:1312-20.

$14 \mathrm{Kim} J \mathrm{E}, \mathrm{Kim} \mathrm{H}$, Choe JY, et al. High expression of sonic hedgehog signaling proteins is related to the favorable outcome, egfr mutation, and lepidic predominant subtype in primary lung adenocarcinoma. Ann Surg Oncol 2013;20(Suppl 3): S570-576

15 Taipale J, Beachy PA. The hedgehog and wnt signalling pathways in cancer. Nature 2001:411:349-54.

16 Finger PT, Albert D, Ainbinder D, et al. Retinoblastoma. In: Edge S, Byrd DR, Compton CC, et al. eds. AJCC cancer staging manual. 7th edn. Chicago, IL: American Joint Committee on Cancer, 2010;561-8.

17 Chantada G, Doz F, Antoneli CB, et al. A proposal for an international retinoblastoma staging system. Pediatric Blood Cancer 2006:47:801-5.

18 Eagle RC Jr. High-risk features and tumor differentiation in retinoblastoma: a retrospective histopathologic study. Arch Pathol Lab Med 2009;133:1203-9.

19 Dimaras H, Coburn B, Pajovic S, et al. Loss of p75 neurotrophin receptor expression accompanies malignant progression to human and murine retinoblastoma. $\mathrm{Mol}$ Carcinog 2006;45:333-43.

20 Griep $A E$, Krawcek J, Lee $D$, et al. Multiple genetic loci modify risk for retinoblastoma in transgenic mice. Invest Ophthalmol Vis Sci 1998;39:2723-32.

21 Hamel W, Westphal M, Shepard HM. Loss in expression of the retinoblastoma gene product in human gliomas is associated with advanced disease. J Neurooncol 1993;16:159-65.

22 Potluri VR, Helson L, Ellsworth RM, et al. Chromosomal abnormalities in human retinoblastoma. A review. Cancer 1986:58:663-71.

23 Squire J, Gallie BL, Phillips RA. A detailed analysis of chromosomal changes in heritable and non-heritable retinoblastoma. Hum Genet 1985;70:291-301.

24 Chan A, Lakshminrusimha $\mathrm{S}$, Heffner $\mathrm{R}$, et al. Histogenesis of retinal dysplasia in trisomy 13. Diagn Pathol 2007;2:48

25 Theunissen JW, de Sauvage FJ. Paracrine hedgehog signaling in cancer. Cancer Res 2009:69:6007-10.

26 Watkins DN, Berman DM, Burkholder SG, et al. Hedgehog signalling within airway epithelial progenitors and in small-cell lung cancer. Nature 2003;422:313-17.

27 Kim J, Kato M, Beachy PA. Gli2 trafficking links hedgehog-dependent activation of smoothened in the primary cilium to transcriptional activation in the nucleus. Proc Natl Acad Sci USA 2009;106:21666-71.

28 Kasper M, Regl G, Frischauf AM, et al. Gli transcription factors: Mediators of oncogenic hedgehog signalling. Eur J Cancer 2006;42:37-445.

29 Wang B, Fallon JF, Beachy PA. Hedgehog-regulated processing of gli3 produces an anterior/posterior repressor gradient in the developing vertebrate limb. Cell 2000;100:423-34.

$30 \mathrm{OK} \mathrm{CY}$, Singh RR, Vega F. Aberrant activation of the hedgehog signaling pathway in malignant hematological neoplasms. Am J Pathol 2012;180:2-11.

31 Mohan A, Kandalam M, Ramkumar HL, et al. Stem cell markers: Abcg2 and mcm2 expression in retinoblastoma. Br J Ophthalmol 2006:90:889-93.

32 Sims-Mourtada J, Izzo JG, Ajani J, et al. Sonic hedgehog promotes multiple drug resistance by regulation of drug transport. Oncogene 2007;26:5674-9.

33 Low JA, de Sauvage FJ. Clinical experience with hedgehog pathway inhibitors. J Clin Oncol 2010;28:5321-6. 